\title{
The effect of ANPR Camera Settings on System Performance
}

\author{
R.Gurney*, M.Rhead*, V.Lyons ${ }^{\dagger}$, S.Ramalingam*, \\ * robert.gurney@ herts.pnn.police.uk, michael.rhead@ @erts.pnn.police.uk, s.ramalingam@ @erts.ac.uk \\ School of Engineering \& Technology, University of Hertfordshire, Hatfield, Herts AL10 9AB, UK \\ ${ }^{\dagger}$ vivienne.lyons@ @omeoffice.gsi.gov.uk, Home Office, Centre for Applied Science \& Technology, Sandridge, Herts. AL4
}

9HQ

Keywords: ANPR, Infra-red, lens, shutter-speed, OCR,

\begin{abstract}
Law Enforcement agencies in the UK have utilised Automatic Number Plate Recognition (ANPR) technology for several decades with a number of high profile crime detection successes. In an effort to ensure that end users are provided with the very best investigative opportunities, data accuracy and system performance must be constantly reviewed. This primary research has been conducted with the aim of better understanding the effect of ANPR camera settings on the performance of imaging systems used by the Police and other Law Enforcement Agencies for crime prevention and detection purposes.

This preliminary research examines key performance factors and outlines empirical real world field assessment of the infra-red set up of a poorly performing fixed ANPR system. The outcomes of this work are intended to support the formulation of international guidance around camera set up published alongside the latest National ANPR Standards for Policing.
\end{abstract}

\section{Introduction}

Automatic Number Plate Recognition technology is already recognised as an incredibly useful technology in the UK and across the world with uses ranging from Traffic Management, Car Park and private land vehicle identification and fuel retail management through to law enforcement and intelligence purposes. There has been a healthy increase in competition for the public sector ANPR market that incorporates Policing, local and national authorities Government agencies.

This expansion has required the solution of a number of issues such as infrastructure and required bandwidth particularly for many of the larger systems Existing manufacturers of ANPR systems have been rightly protective of their proprietary installation and set-up knowledge.

Some systems use existing CCTV cameras to provide ANPR data as this technology falls well within their scope of operational knowledge. However it is essential that there is an understanding of the differences between them and how they may be used to fulfill ANPR requirements.
There are a number of challenges that face ANPR installers ranging from available broadband width, locality of power supply through to environmental conditions such as problems with local geography and weather. However, some of these factors can be mitigated by appropriate camera set-up from the outset.

The first two authors have been involved in assessing ANPR systems in real world situations over a number of years. These assessments have produced a number of findings ranging from some very accurate systems where time and care have been spent during the initial set up process through to some woefully inadequate systems installations where the installer has signed off the work purely based on their own "looks ok to me" principle.

In summary the positioning and set up of an ANPR camera plays an incredibly important part in the capture process and the outcomes of this work will provide some generic guidance to those involved in the installation and setup process, either installers or customers requiring optimum returns on investment. This work follows on from previous research into the real world accuracy of ANPR systems carried out on behalf of the Police Service nationally. [1] [2] [3]

Section 2 outlines the primary factors affecting camera performance. Section 3 describes the experimental setup to establish camera performance and the field testing process. Section 4 discusses findings and Section 5 provides a conclusion and proposals for further work.

\section{Performance Factors}

This research followed previous field work that has clearly demonstrated a link between misreads (wrongly interpreted registration marks) and missed reads (registration marks which are not captured) with both poor number plate quality and improper camera setup.

It is however important to note that camera set up does not form part of the current policing ANPR standards [4] [5]. As previously mentioned the compliance testing assumes that the camera has been correctly set up and reports on activity within the camera's field of view.

Many users of the current national standard refer to it as an "ideal world" test and there is a huge drive from chief 
police officers for an improvement in the accuracy of data obtained from ANPR systems. There is an operational requirement for the best possible accuracy in an effort to detect crime. Also there is pressure from the various information security commissioners whose role is to protect personal data belonging to the public.

Misreads are an important factor to consider when trying to ascertain the causes of poor data quality. Over many years, the authors have spent many hours reviewing real-world data in an effort to establish the reasons for both missed and misread data. There are a variety of factors that can cause difficulties to the Optical Character Recognition (OCR) system that is ultimately responsible for determining the correct number plate. This point is illustrated more clearly by looking at the following figure.

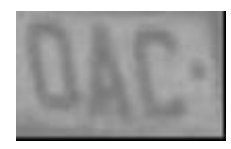

Fig.1: Image discounted from testing of NAAS compliancy

In this image we can see a number of difficulties that may be presented to the OCR system that has led to the image itself being unclear. This may be due to a number of reasons such as a dirty number plate, insufficient illumination (natural light or infrared), incorrect manufacture, or the camera may not have been set up correctly.

Whatever the cause of the misread resulting from a poor image, the outcome is causes problems for the end user. For a police officer it may result in both false positives where a misread can result in an incorrect "hit" on a hotlist resulting in wasted time for all involved. Conversely, it may result in a false negative, where the vehicle of interest is in fact not picked up at all because its number plate has been read incorrectly. The term "hit" describes a match between a vehicle registration number image capture obtained by an ANPR camera and a number held on a system database.

This research has concentrated on camera setup and deployment and it is clear that the physical properties of the camera form an integral part of the data acquisition process. There are a number of relevant factors that should be taken into consideration when considering this work, such as:

- Iris size

- Shutter speed

- Positioning of camera

- Height

- Angle

- Range of view

- On-board processing capability

- Lighting/wavelengths

- IR illuminators

- Type and size of lens

\subsection{Camera Settings}

All ANPR systems rely on OCR to analyse the images that are captured by cameras that are linked to the system. It necessarily follows that higher accuracy results from better quality input images. It is important to note that better detail is difficult to obtain from a blurry image. Accordingly, from the outset an image with useful sharpness and contrast must be acquired if the system is going to have the best chance of interpreting number plate data correctly and reliably. In order to achieve this we need to ensure that the following camera specific factors are combined in the right way:

- Optics

- image sensor

- camera

- lighting

\subsection{Achieving good quality ANPR images}

Accurate identification of the plate within the captured image is often considered to be the initial stage of the recognition process. There are many factors that cause difficulty with this including vehicles moving between lanes, number plates that are not being displayed in the correct manner (e.g. falling off), obscuration caused by queuing traffic etc.

There are a number of key elements that contribute to producing an accurate and useful image. These can be described as follows:

\subsubsection{Camera Alignment}

It is essential that the camera is set up to meet the requirements of the user, which is generally one lane. Some manufacturers produce HD cameras that will cover more than one lane but it is important to note that resolution per lane will normally be reduced. This can be problematic if reading number plates of vehicles travelling in two directions since front and rear number plates have differing reflective capabilities.

There are a wide range conditions for cameras to cope with including traffic speed traffic flow rate, number plate location and capture range. The angle of alignment is equally important and is dictated by a number of factors, i.e. camera height, road layout and vehicle positioning. This aspect is discussed later in this paper. ANPR cameras perform optimally when they are perpendicular to and level with the number plate. All installations, whether they are on motorway bridges or purpose built columns will not be perpendicular to the number plate. Camera software has to take account of declination and skew in the positioning of the camera with respect to the number plate when processing images. Likewise adjustments may be necessary where ANPR cameras are positioned close to a bend or roundabout or where temporary road works mean that traffic is diverted between standard lanes.

\subsubsection{Sharpness}

Sharpness or focus characterises the clarity of the image and is one of the most important factors in establishing image quality. Fine details will be lost in the blurring of spatial detail, something that can easily occur within ANPR systems 
especially those that are deployed in areas where vehicles travelling at high speed such as motorways or dual carriageways.

\subsubsection{Depth of field}

This is commonly defined as being the distance between the nearest and farthest objects in the image that appear acceptably sharp. ANPR images need to be sharp across the whole image; hence a large depth of field is required. This can be achieved by using small, rather than larger, iris openings and there are several ways of compensating for the resultant reduction in image brightness. A more sensitive sensor can be used and/or a lens with a lower " $F$ " number could be used. Care is needed in the latter case because fast, low "F" number lenses have smaller tolerances for the optimum focus setting.

\subsubsection{Motion Blur}

Motion blur is a common term used to describe the image that often results trying to image a fast moving object. This problem is often encountered by ANPR systems operating in high-speed environments and is more commonly experienced in mobile vehicle borne ANPR systems.

The problem can be mitigated through shorter exposure times and this can be achieved in several ways. Firstly if more sensitive sensors are used, less light is required to capture a good image with shorter exposure times. This is particularly important as it allows better images to be captured of moving vehicles. Secondly, shorter exposure times can also be achieved by using a lower "F" value lens which allows more light to be captured with each exposure.

\subsubsection{Lighting}

The authors' previous work on reflectivity highlighted the fact that number plates have a variety of reflection coefficients and the wavelength of visible or infrared lighting required will very much depend on the country in which the ANPR system is being deployed. Even in the UK, where there is a British standard applicable to the manufacture of number plates, a variety of visible and infrared wavelengths are deployed.

The level of light available to ANPR systems is an extremely important factor in determining performance and requires assessment and set up for each individual system. Illumination is available from a variety of sources ranging from natural sunlight through to artificial light created by street lighting, headlamps or, as commonly found in the UK. Most ANPR cameras have their own specially designed infrared illuminators. In order to ensure optimum performance it is important to ensure that the correct levels of light reach the whole of the camera field of view. If there are areas of the capture zone with insufficient or excessive light, OCR systems may have difficulty interpreting the image and misreads may occur.

Some systems rely on ambient lighting (daylight) with added street lighting during the hours of darkness, but this does present problems. If the "ambient" light source is lost (e.g. blown street lamp), capability is lost. Excess light caused by a car flashing main beams or switching to main beam can produce capture issues from excessive light.

Other systems are designed to rely on inbuilt infrared light sources. These systems work well with ambient light filters which can reduce the effects of bright sun and headlamp glare. It is important to consider the considerable amount of infrared in natural sunlight as this too can affect the success of the ANPR system.

\subsubsection{Iris Control}

Although automatic Iris control is widely available, it is not often popular with ANPR manufacturers because a fixed Iris offers much more control over the focus and exposure.

A work-around can be achieved by taking multiple images of the same object with a variety of exposure times with the fixed iris.

\subsection{Contrast}

Contrast is one of the most important elements of the OCR process and is key to accurate data interpretation. It is perhaps best described as the difference between the light and dark areas in an image. Pronounced differences will enable much better character recognition and the authors' work on reflectivity has examined the effects of both good and bad contrast. In essence, a strong contrast between number plate characters and background will provide optimum interpretation opportunities.

\subsubsection{Improving contrast in ANPR systems}

There are a number of ways that contrast can be improved and these include optimal lighting, using cameras with higher frame rates, using HD cameras and cameras with large dynamic range.

\subsubsection{Lighting}

Matching the illumination including IR wavelength with number plates according to the environment will assist in achieving optimum read rates. Contrast can be extremely limited if there is poor reflection of light on the retroreflecting background of the number plate. Whilst, there is a British standard that states the minimum visible light number plate coefficient of retro reflection the authors' research has established that there is a wide variation in the retro reflection displayed by UK number plates, particularly between visible and IR illumination. Environmental factors such as daylight vs. darkness, weather variations from bright sunlight to snow, rain and fog all have a great impact on the lighting levels required.

\subsubsection{Higher Frame rates}

All ANPR systems utilise cameras with high frame rates that enable numerous image captures to be obtained using a 
variety of exposure times. The great benefit of doing this is that the very best image of a number plate can then be selected before being passed through the OCR system.

\subsubsection{HDR Imaging}

Cameras with High Dynamic Range (HDR) have proved popular with some ANPR manufacturers as their image sensors enable better differentiation between characters and backing plates. Optimum conditions are achieved by ensuring that the full linear dynamic range of the sensor is available and this is particularly useful in countries such as the USA where number plate legislation and make-up of the plate varies from state to state and India where a host of different number plate styles are in operation.

Non-HDR cameras capture images at a single exposure level with a limited contrast range and this very often culminates in detail loss in parts of an image. With HDR imaging the problem is overcome by taking many photographs at a variety of exposure levels and then combining them to create an image that is representative in all areas both bright and dark.

\subsection{Minimising the effect of image noise}

Reducing the occurrence of image sensor artefacts is challenging but appropriate engagement with camera manufacturers can lead to their removal or reduction. There are a number of factors that contribute to artefacts being seen in images which can confuse an OCR algorithm and produce misreads. Fortunately there are ways of mitigating their effect on ANPR systems and in particular OCR.

\subsubsection{Streaks and blooming}

Streaks and blooming are problems that need to be overcome in many external camera systems including ANPR. They are often caused by saturation from very bright areas within a captured image. Clearly such artefacts can have a very negative effect upon the ability of systems to identify objects within the image such as number plates.

Common examples of this type of saturation can originate from bright sunshine, reflections off of the road surface, and variations in the position of the sun throughout the year, reflections from number plates and light from vehicle headlamps. The OCR parts of ANPR systems are not generally able to counter the effects that they produce. These problems may be solved by adjustments to the camera or its functionality.

\subsubsection{Ghost images}

In some circumstances, ghost images can appear in a scene particularly where infrared lighting is used combined with a high pass filter. This was shown when the authors conducted research previously where such a filter was fitted to a standard camera to produce images within the infrared spectrum. Ghost images can be prevented through the use of a filter that is optimum for the IR wavelength used, the camera and the lens.

\subsection{Environmental factors \& site considerations}

No two installation sites are the same; there are always a variety of environmental factors that should be taken into consideration. These are likely to include but are not limited to the following:

- Type of installation: permanent, temporary or mobile

- Weather can have a huge impact on ANPR performance and inclement conditions such as snow, hail, rain and sandstorms not only obscure camera views but they can also physically obscure a number plate. Different settings and camera shields may be required according to individual circumstances.

- Foliage, trees and street furniture that may impede the view of the camera

- Physical positioning according to site: on motorways this is likely to be high up on a gantry or bridge, on A roads this will often be high up beside the road and at barrier controls this may be at headlamp height

\section{Field Assessment}

The authors reviewed capture rates for a large number of ANPR cameras and found that capture rates for many cameras were worse during the evening rush hour in October than they were during June. Some cameras gave a reduction in capture rate of between 5 and $60 \%$.

A number of possible reasons for the differences were considered. A review of infrared videos from some ANPR cameras indicated that some number plates failed to be captured because of insufficient infrared illumination.

One poorly performing static camera was tested to see if this supported the view that the capture point had been set outside the infrared illumination range.

\subsection{Initial Field Testing}

A fixed Police ANPR camera had been set up and tested according to NAAS [4] by an installation contractor. A drop in performance was identified by Mike Rhead, an authorised Systems Analyst during a regular review of the local ANPR database. Subsequent reviews of misreads and site visits clearly indicated that the camera had been incorrectly set up which was then confirmed by the camera manufacturer. In order to identify setup issues the authors set about carrying out some further research.

An initial assessment was conducted by parking an ANPR equipped Police car just beyond the target ANPR camera. The intent was to capture read data from the Police car system and compare it with data obtained from the "underperforming" fixed camera. The authors compared the video recording from the police vehicle with the ANPR camera's captures to find any discrepancies. 
This data was also compared to results from a fixed ANPR camera located approximately one quarter of a mile away. This camera was also used to ascertain read rates and any disparity between the two cameras. It soon became apparent that the target camera was performing worse during the hours of darkness.

\subsection{Testing Methodology:}

The initial measurements had shown differences between daylight and darkness read rates had already been identified and a night time vehicle comparison had likewise been conducted. It was agreed that capture zones needed to be identified and these would require roadside measurements to be taken. To avoid health and safety issues it was decided to take primary data measurements during daylight hours. This also provided less opportunity to arise for other factors to influence the results, for example number plate reflectivity at night time, the potential for headlamp interference etc. The test could easily be replicated at night time if this was later thought necessary but the initial aim was to ascertain capture zones and both day and night read rates.

\subsection{Initial Findings:}

As this camera was setup according to the previous standard a subsequent review of the company's NAAS calculations indicated that the installer had misunderstood the requirements of NAAS. Their figures showed a $95 \%$ capture rate and a $98 \%$ correct read rate which according to the contractor which gave the required $93 \%$ overall efficiency. This was the reverse of the actual capture and read rate requirement. The researchers pointed out their error both to them and to the UK Policing ANPR community so that they got a better understanding of some of the inaccurate data that is sometimes being presented. In this case ANPR camera setup guidelines were prepared by the researchers, which the contractors stated that they had followed in readjusting all of their cameras.

The researchers subsequently carried out further field assessment tests on the camera during both daylight and darkness. They quickly established that the capture rate proved to be far too low for a NAAS compliant system and a further review of the results indicated that the camera's missed captures and misread rate were both unsatisfactory and they were subsequently improved.

The latest standard has redefined the minimum requirements for both capture rate and correct read rate.

\subsection{Capture zone settings:}

A vital factor in setting up an ANPR system which performs well is ensuring that the capture zone i.e. the optimum distance from the camera to the target vehicle for the infrared illumination used, is properly calculated. The factors that must be taken into consideration are camera height, infra-red power/range of the camera. One must also consider that the start of a capture zone will be an arc from the camera rather than a straight line across the road from the pavement. This is demonstrated in figure 1 . This is especially relevant on a dual or three lane carriageway as the actual capture zone in the form of an arc will be much less than the perceived (or pavement measured) line.

At the particular installation under investigation the start of the capture zone should have been set to start at 23 meters whereas it has been set at between 30 and 33 meters. Clearly there would be a huge misread / missed read concern and the camera was performing far worse than would reasonably have been expected in that location.

The second author created a guidance document for the contractors based on the arc capture zone which they have followed and our subsequent assessment has shown a significant increase in read rates.

\subsection{Experimental detail}

The ANPR camera concerned has an operational range of 6$25 \mathrm{~m}$ and the camera manufacturer recommends setting the camera such that the capture of a vehicle is in the range 18-23 metres for the camera type tested. The figures in appendix A represent capture zones.

Figure 1 is a schematic layout of one camera installation for a two lane highway. This installation was tested by a series of known vehicles driving past the camera on multiple occasions, both day and night, using both lanes. The camera gave some misreads for both lanes and a substantial number of missed reads at night for lane 1 (the lane furthest from the camera).

Figure 1 indicates where three cones were placed; $18 \mathrm{~m}$, $23 \mathrm{~m}$ and at $32 \mathrm{~m}$. These distances are represented by green, red and black lines respectively. The figure also indicates the $18 \mathrm{~m}$ and $23 \mathrm{~m}$ arcs across the road. The grey shaded area represents the capture zone which is $18 \mathrm{~m}$ to $23 \mathrm{~m}$ from the camera.

Figure 2 gives examples where the vehicles are captured outside of the recommended range. There is likely to be insufficient infrared illumination at this range from the cameras illuminators.

Figures 3 and 4 give examples where the vehicles are captured at the limit of the recommended range. It is is likely that there will be sufficient infrared illumination at this range from the cameras illuminators.

Figures 5 and 6 give examples of captures from the revised setup methodology. The revised methodology required the technicians to measure, (and clearly mark), the $23 \mathrm{~m}$ and $18 \mathrm{~m}$ points. The camera was then set to capture vehicles within the marked points.

As can be seen from figures 5 and 6 vehicles are captured within the specified zone and within the infrared illuminator range. Subsequent testing of these cameras indicated no misreads or missed reads for our convoy's multiple passes both day and night.

The camera under investigations is capable of monitoring two lanes of traffic providing the width is not more than about 6 metres. To achieve this then the field of view will need to clip 1 metre from the edge of the nearside lane and from the edge of the outside lane. This should result in no missed 
vehicles unless they drive on the pavement or central reservation to go past the camera.

\section{Discussion}

This research has shown that that correct camera installation set up and on-going monitoring is critical to achieving optimum read rates.

To the untrained eye, the assessment of ANPR cameras may appear deceptively easy. In practice this involves looking at a number of parameters from height/distance/vehicle frequency through to IR wavelength. Some of these variables might be difficult to measure due to practical problems. Manufacturers have stated that a number of these parameters either cannot be measured or are not commercially and practically viable to measure and as such become the unknowns in the experiment. A good example of a difficult parameter to measure is illuminator wavelength of cameras mounted high on fixed poles.

\section{Conclusion and Further Work}

High quality input images provide better opportunities for OCR which in turn results in better read accuracy. The challenges of image artefacts can be overcome by ensuring that the camera lens, the camera, the filter and lighting are properly aligned. By combining this alignment with optimal contrast and sharpness very high quality images can be obtained. This in turn enables the OCR software to work more efficiently leading to much better overall performance.

The authors recommend further research into camera setup to ensure optimum performance is achieved both at installation and throughout the life of the system.

\section{Appendix A}

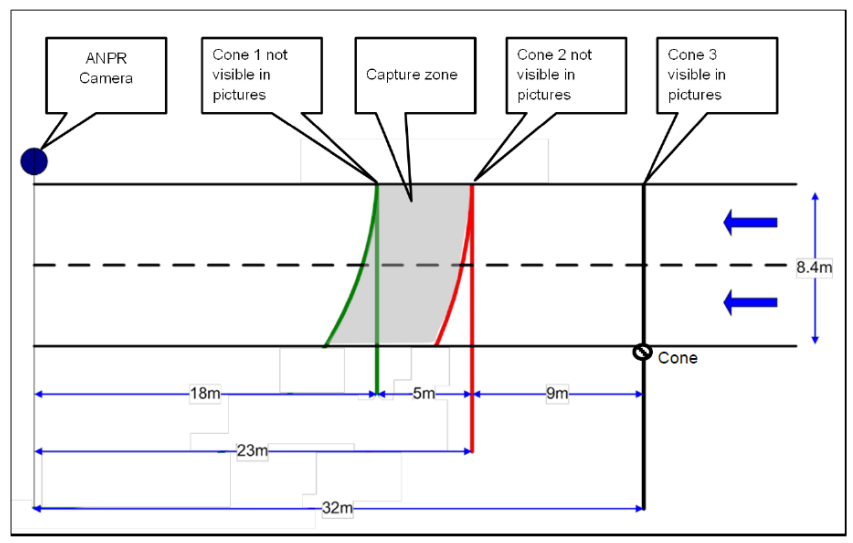

Figure 1 ANPR Camera A testing schematic

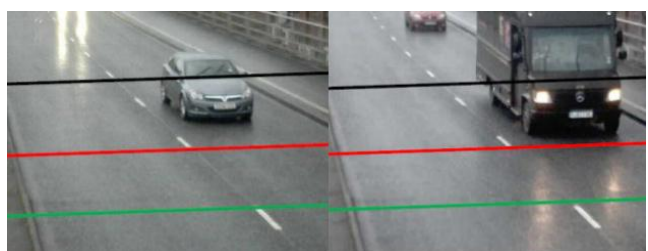

Figure 2 ANPR Camera A captured too early

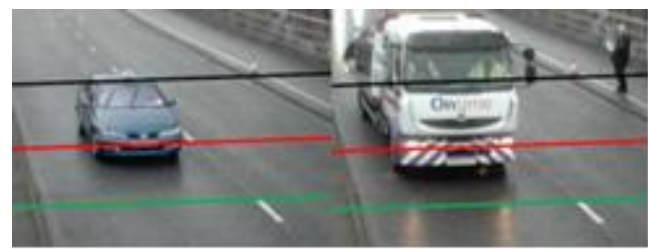

Figure 3 ANPR Camera A examples of being captured at about the maximum recommended range

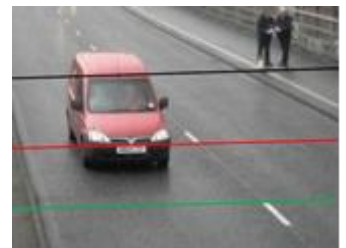

Figure 4 ANPR Camera A being captured at $23 \mathrm{~m}$

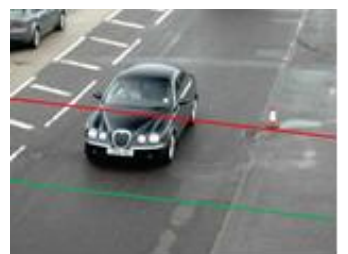

Figure 5 ANPR Camera B

Captured between $18-23 \mathrm{~m}$

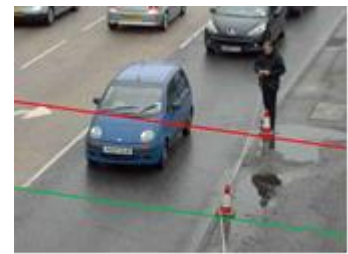

Figure 6 ANPR Camera C Captured between 18-23m

\section{Acknowledgements}

Dr Peter Billington of UTMC deserves special credit for the valuable contribution that he has made to support this research. Mark Jones of the Home Office and Detective Superintendent Paul Ealham of the ACPO ANPR Countermeasures Group warrant special mention for the strategic support that they have given to this work.

\section{References}

[1] M. Rhead, R. Gurney, N. Cohen, S. Ramalingam, Accuracy of Automatic Number Plate Recognition (ANPR) and Real World UK Number Plate Problems, IEEE International Conference on Security Technology, 15-18 Oct. 2012, Boston, Massachusetts, USA.

[ 2] R. Gurney, M. Rhead S. Ramalingam, N. Cohen, Working Towards an International Automatic Number Plate Recognition (ANPR) Standard - An Initial Investigation into the UK Standard, IEEE International Conference on Security Technology, 15-18 Oct. 2012, Boston, Massachusetts, USA.

[3] M. Rhead, R. Gurney, S. Ramalingam, N. Cohen, Impact of UK Number Plates on the Accuracy of Automatic Number Plate Recognition (ANPR), Journal of Information Assurance and Security Letters, http://www.mirlabs.net/iasl/, accepted and revised submitted, Aug. 2012.

[4] The National ACPO ANPR Standards (NAAS) National Policing Improvement Agency 2008

[5] National ACPO ANPR Standards for Policing (NASP) Proposed August 2013 